\title{
Collective Excitations, NMR, and Phase Transitions in Skyrme Crystals
}

\author{
R. Côté, ${ }^{1}$ A. H. MacDonald, ${ }^{2}$ Luis Brey, ${ }^{3}$ H. A. Fertig, ${ }^{4}$ S. M. Girvin, ${ }^{2}$ and H. T. C. Stoof ${ }^{5}$ \\ ${ }^{1}$ Département de Physique,Université de Sherbrooke, Sherbrooke, Québec, Canada J1K-2RI \\ ${ }^{2}$ Department of Physics, Indiana University, Bloomington, Indiana 47405 \\ ${ }^{3}$ Instituto de Ciencia de Materiales (CSIC),Universidad Autónoma C-12, 28049 Madrid, Spain \\ ${ }^{4}$ Department of Physics and Astronomy, University of Kentucky, Lexington, Kentucky 40506-0055 \\ ${ }^{5}$ Institute for Theoretical Physics, University of Utrecht, P.O. Box 80.006, 3508 TA Utrecht, The Netherlands
}

(Received 21 February 1997)

At Landau level filling factors near $\nu=1$, quantum Hall ferromagnets form a Skyrme crystal state with quasi-long-range translational and noncollinear magnetic order. We develop an effective low energy theory which explains the presence of magnetic excitations in these systems at energies below the Larmor gap $(\Delta)$ and that predicts a dramatic enhancement of the nuclear spin relaxation rate by a factor of $10^{3}$. The effective theory predicts a rich set of quantum and classical phase transitions. Based in part on accurate time-dependent Hartree-Fock calculations of the ordered state collective excitation spectrum, we discuss aspects of the $T-\nu-\Delta$ Skyrme crystal phase diagram. [S0031-9007(97)03424-8]

PACS numbers: 73.40.Hm, 64.60.Cn, 75.10.-b

At Landau level filling factor $\nu=1 / m$ (with $m$ an odd integer), the ground state of a two-dimensional electron system (2DES) is an incompressible strong ferromagnet [1], i.e., it has complete spin alignment even in the limit of vanishing Zeeman coupling strength and it has a gap for charged excitations. Quantum Hall ferromagnets have the unusual property [2-4] that their topologically nontrivial spin texture excitations (Skyrmions) carry charge. This property profoundly affects their physics. In particular, the ground state at filling factors slightly away from $\nu=1 / m$ contains a finite density of Skyrmions, and these are expected [5-7] to crystallize, at least when they are sufficiently dilute.

In an external field the spin-wave modes of a collinear ferromagnet have an excitation gap equal to the Zeeman energy. On the other hand, a noncollinear magnet can have two Goldstone modes [8], and one of these can remain gapless in an external field. The Skyrme crystal state has noncollinear magnetic order [5]. A single Skyrmion spin texture has its spins aligned with the Zeeman field at infinity, reversed at the center of the Skyrmion, and has nonzero $X Y$ spin components at intermediate distances which have a vortex-like configuration [2,6]. The classical (or quantum mean-field) energy of a Skyrmion is independent of the angle $\varphi$ which defines the global orientation of the $X Y$ spin components. This extra $U(1)$ degree of freedom for a single Skyrmion leads to broken symmetry in the crystal ground state and hence to a spin wave mode which remains gapless in the presence of the Zeeman field. The existence of this gapless spin mode (presumably in overdamped form in a Skyrme liquid state) dramatically alters the low temperature physics, manifesting itself in both rapid nuclear spin relaxation [9] and giant apparent specific heat [10].

In this Letter we address the impact of thermal and quantum fluctuations on the physics of Skyrme crystals. We propose a rich zero temperature phase diagram in which quantum fluctuations destroy the magnetic order of the Skyrme crystal for some values of $\nu$ and Zeeman coupling strength. Where order exists in the ground state, we estimate the finite temperature Kosterlitz-Thouless phase transition temperatures. Our analysis is based on a boson Hubbard model, which we argue describes the low-energy physics of this system, and on time-dependent Hartree-Fock approximation (TDHFA) calculations of the collective modes of the Skyrme crystal, which accurately capture the intricate microscopic physics of the crystal state and fix the Hubbard model parameters.

The simplest symmetry-allowed orientation-dependent coupling between neighboring Skyrmions is of the form $V=J \sum_{\langle i j\rangle} \cos \left(\varphi_{i}-\varphi_{j}\right)$. For typical Zeeman coupling strengths and $\nu$ not too close to 1, detailed HartreeFock calculations [5] show that the Skyrme crystal is a square lattice with opposing orientations $\left(\varphi_{i}=\varphi_{j}+\right.$ $\pi)$ for Skyrmions on opposite sublattices. The lowenergy effective Hamiltonian we propose is motivated by microscopic theory from which it follows [11] that, in addition to the positional degrees of freedom, Skyrmions carry an integer-valued internal quantum number $(K)$ which is the number of flipped spins (relative to the maximally polarized state of the same charge). Quantum states of definite $K$ are related to the classical states of definite orientation by $[11,12]|K\rangle \propto \int d \varphi \exp (i K \varphi)|\varphi\rangle$, so that for each skyrmion $K_{i}$ and $\varphi_{i}$ are canonically conjugate. Thus our effective model is equivalent to a boson Hubbard model [13] in which the boson number on the $i$ th site is mapped to the number of flipped spins in the $i$ th skyrmion. In this language the orientation-dependent interaction term corresponds to boson hopping and favors long range boson coherence.

We limit our attention for the moment to states where the Skyrmions have crystallized. At low energies, phonon excitations of the lattice decouple [14] from the spins and can be treated separately. Because of the Larmor gap and the incompressibility gap for particle-hole excitations, 
fluctuations in $K$ are the only relevant low-energy degrees of freedom, if the Skyrmion positions are fixed. The energy of an isolated Skyrmion in state $K_{i}$ can be written in the form [6] $\epsilon_{K_{i}}=U_{K_{i}}+g^{*} \mu_{B} B K_{i}$, where $g^{*}$ is the host semiconductor $g$ factor. Nonlinearity in the function $U(K)$ maps to on-site boson interactions. In the Zeeman coupling term on the right-hand side of this equation, $-\tilde{g} \equiv-g^{*} \mu_{B} B$ plays the role of a chemical potential for the bosons. (In typical experimental systems $\tilde{g} \sim 0.015\left(e^{2} / \epsilon \ell\right)$.) Making a quadratic approximation for $U_{K}$, we are led to an effective Hamiltonian describing boson fluctuations for states with $K$ near $\langle\hat{K}\rangle \equiv K^{0}(\tilde{g}, \nu)$ :

$$
H=U \sum_{i}\left(\hat{K}_{i}-K^{0}\right)^{2}+J \sum_{\langle i j\rangle} \cos \left(\varphi_{i}-\varphi_{j}\right) .
$$

For $J \gg U$, boson hopping dominates over interactions so that the bosons condense into a supersolid phase with long range phase coherence. A linear spin wave approximation for this model yields a gapless Goldstone mode with dispersion

$$
E(\mathbf{k})=\left\{4 U J\left[2-\cos \left(k_{x} a\right)-\cos \left(k_{y} a\right)\right]\right\}^{1 / 2} .
$$

However, unless $2 K^{0}$ is an odd integer, quantum fluctuations will destroy the classical ordered state and produce a gapped "insulating" state [13] when $U \gg J$.

The parameters of this model can be fixed using microscopic Hartree-Fock calculations [5]. From the ground state calculation we can evaluate the number of reversed spins per charge, $\langle\hat{K}\rangle$. In the harmonic approximation, the boson interaction parameter $U$ is given by the inverse boson compressibility:

$$
U=-\frac{1}{2}\left(\frac{\partial\langle K(\tilde{g}, \nu)\rangle}{\partial \tilde{g}}\right)^{-1} .
$$

The boson hopping parameter $J$ can then be extracted from the collective mode spectrum which we compute by locating poles of the time-dependent Hartree-Fock approximation (TDHFA) spin and density response functions, $\chi_{\mu \nu}\left(\mathbf{k}+\mathbf{G}, \mathbf{k}+\mathbf{G}^{\prime}, \omega\right)$. The TDHFA calculation is facilitated by simplifications which arise from the restricted Hilbert space of the lowest Landau level $[14,15]$. The collective mode spectrum in the extended BZ of the square lattice Skyrme crystal is illustrated in Fig. 1. The two gapless modes are the phonon mode and the new spin-wave collective mode discussed above. The dashed lines show the same modes folded into the smaller BZ of the magnetic lattice. As $k \rightarrow 0$, the higher energy of the two-zone folded modes evolves into the Larmor mode (global precession of electronic spins around the Zeeman axis) which must occur at $\hbar \omega_{0}=g^{*} \mu_{B} B$. The fact that the (new) spin wave mode at $\mathbf{k}$ equal to a reciprocal lattice vector of the magnetic lattice is equivalent to the $\mathbf{k}=0$ Larmor mode can be understood in terms of the opposite orientations of Skyrmions on the two sublattices [14]. Because of the inhomogeneous magnetic order of the Skyrme crystal state, spin and density response func-

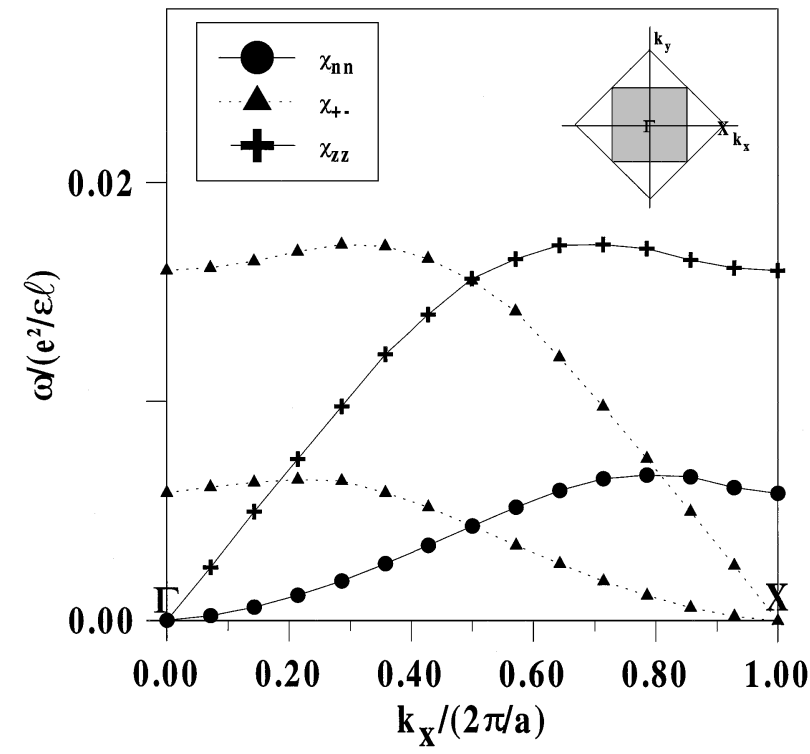

FIG. 1. Collective mode energies in $e^{2} /(\epsilon \ell)$ units for $\nu=$ $1.10, \tilde{g}=0.016\left(e^{2} / \epsilon \ell\right)$, along the direction $\Gamma-X$. The shaded region is the magnetic Brillouin zone. At each $\mathbf{k}$ a mode is labeled by the response function component with the largest residue in $\chi(\mathbf{k}, \mathbf{k})$.

tions couple at general points in the BZ, and poles occur in all response functions at each collective mode. Figure 1 confirms that at low energies the only relevant degrees of freedom are skyrmion orientations and locations, and that these give rise to well separated spin-wave and phonon modes. As $k \rightarrow 0$, the phonon mode is lowest in energy and has the characteristic $k^{3 / 2}$ magnetophonon dispersion. We estimate $J$ by requiring Eq. (2) to reproduce the spin-wave velocity of the microscopic TDHFA calculations. In Fig. 2 we plot the boson Hubbard model parameters $\left(K^{0}, U\right.$, and $\left.J\right)$ for square lattice Skyrme crystals at a series of $\tilde{g}$ values and for filling factors $\nu=1.05$, $\nu=1.1, \nu=1.15$, and $\nu=1.2$.

Even the $T=0, \nu-\tilde{g}$ phase diagram of the Skyrme crystal states is very rich. Figure 3 summarizes conclusions (some still qualitative) we have drawn from present calculations. The line of primary importance in this phase diagram is the dark solid line which delimits [16] the stability region of the square lattice. In our calculations the instability of the square lattice is indicated by vanishing long wavelength magnetophonon energies. The square lattice shear constant is negative for classical electrons, and this structure is therefore expected [14] to become unstable when Skyrmion orientation-dependent interactions become weak. The square lattice is stable in the small $\tilde{g}$, large $|\nu-1|$ region where the Skyrmion size is comparable to the crystal lattice constant. At low Skyrmion density, the critical value of $\tilde{g}$ is expected to vary as $|\nu-1|^{3 / 2}$, corresponding [14] to a fixed ratio of Skyrmion size to Skyrme crystal lattice constant. We have not yet completed a systematic survey of possible crystal structures outside this region where, in 


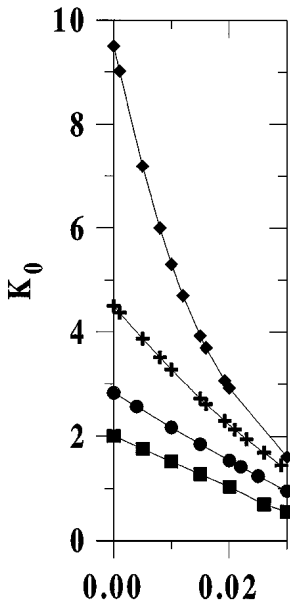

$\tilde{\mathbf{g}}$

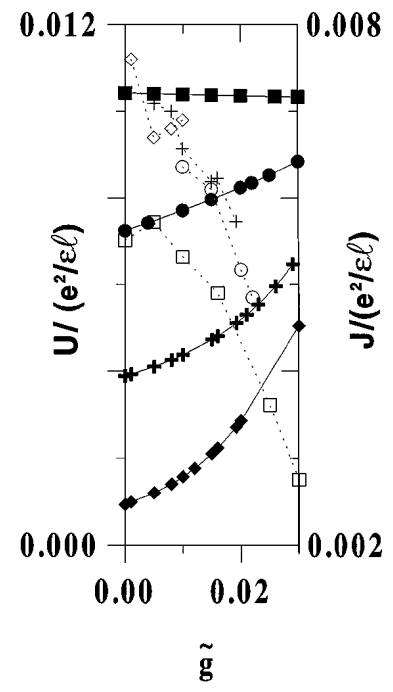

FIG. 2. Parameters of the low energy effective model for $\nu=1.05$ (diamonds), $\nu=1.1$ (plusses), $\nu=1.15$ (circles), and $\nu=1.2$ (squares) as a function of $\tilde{g} /\left(e^{2} / \epsilon \ell\right)$. In the right panel the solid symbols give values of the boson interaction parameter $U$ while the open symbols give values of the boson hopping parameter $J$. The noise in the latter curve reflects numerical uncertainty introduced by the fitting procedure used to extract the spin-wave velocity from calculated collective mode energies.

any event, Skyrmion-disorder interactions will frequently play a larger role than orientation-dependent SkyrmionSkyrmion interactions. Inside the square Skyrme crystal portion of the diagram, our estimates place $J / U>$ 1 except at large $\nu-1$. We therefore expect to have a ground state with translational and magnetic order in the shaded portion of the phase diagram, which excludes regions where $K^{0}$ is near an integer, $J / U<1$, and we expect magnetic-order to be destroyed by quantum fluctuations. This phase diagram is subject to quantitative alteration when quantum fluctuations in the Skyrmion positions become large at large $|\nu-1|$. Outside of the squarelattice portion of the phase diagram, Skyrmion orientations will be more weakly coupled and we expect quasi-longrange magnetic order to be rare.

We can extract a rough upper bound for the KosterlitzThouless critical temperature associated with the loss of quasi-long-range magnetic order: $T_{\mathrm{KT}}=\left(\pi / 2 k_{B}\right) \rho_{s} \sim$ $0.008\left(e^{2} / \epsilon \ell\right) \sim 1 \mathrm{~K}$. Here $\rho_{s}=J$ is the spin stiffness for the classical $X Y$ model obtained when $U=0$ and the numerical estimate is for typical magnetic fields $\sim 10 \mathrm{~T}$. This temperature should be compared with the classical melting temperature of the crystal which can be estimated using KTHNY [17] theory. For elastically isotropic systems with long-range interactions, $T_{M}=\left(b^{2} \mu / 4 \pi\right)$ where $\mu$ is the shear constant and $b$ the lattice constant of the crystal. Neglecting the elastic anisotropy of the square lattice $[14,18]$, we estimate $\mu$ for the Skyrme crystal from the long wavelength dispersion of the magnetophonon mode $\hbar \omega(k) \approx\left(2 \pi \mu \ell e^{2} / \epsilon\right)^{1 / 2}(k \ell)^{3 / 2}$. These considera-

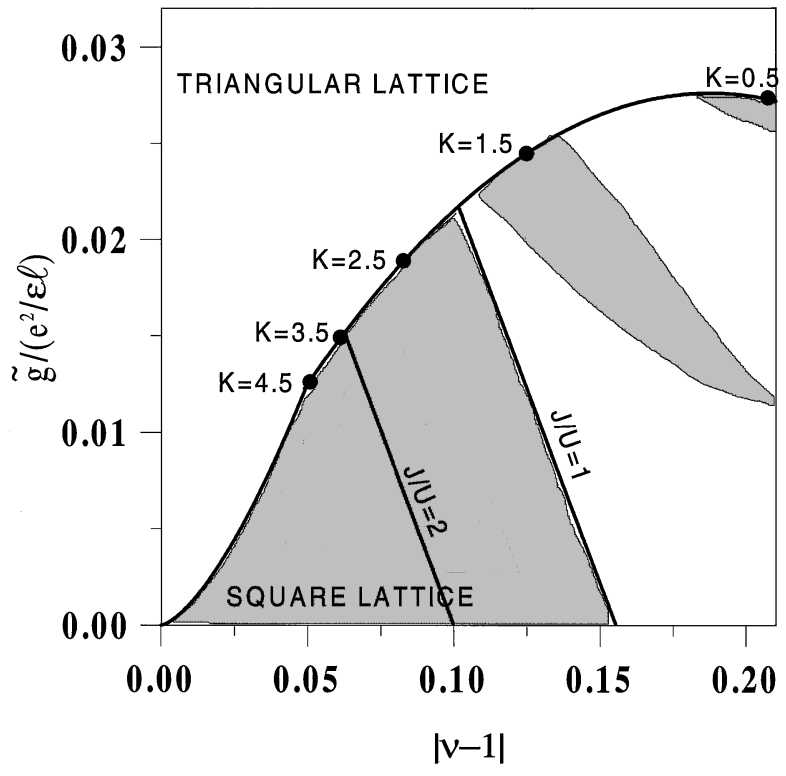

FIG. 3. $T=0$ phase diagram for Skyrme crystal states. The value of $\tilde{g}$ at which the square lattice shear modulus vanishes has been determined numerically at $|\nu-1|=0.05,0.1,0.15$, and 0.2 and interpolated to delimit the square lattice stability region. The solid circles indicate the variation of $K_{0}=$ $\langle K\rangle$ along the square lattice region boundary. Noncollinear magnetic order survives quantum fluctuations in the region (approximately) indicated by the shading.

tions lead to melting temperatures at $\tilde{g}=0.015\left(e^{2} / \epsilon \ell\right)$ of $5.23,3.63$, and 2.51 [in units of $10^{-3}\left(e^{2} / \epsilon \ell\right) \sim 0.1$ $\mathrm{K}]$ for $\nu=1.10,1.15$, and 1.20, respectively. Therefore, we expect that the melting transition will occur at a lower temperature than the magnetic transition. Dislocations in the "tetratic" fluid state frustrate the Skyrmion orientational order, so the spins presumably disorder at the same temperature in a single transition [14]. For classical electrons the melting temperature of the electron crystal is lower than the KTHNY estimate by a factor of $\sim 2$; quantum positional fluctuations [19] cause a further reduction. Since orientational order is responsible for the positive $T=0$ shear modulus of the square Skyrme crystal, thermal and quantum fluctuations of the $X Y$ order are likely to lead to an even larger reduction in the present case.

At $\nu=1$, spin-relaxation in a quantum Hall ferromagnet is activated because of the Larmor mode gap. For $|\nu-1| \neq 0$, however, both phonon and spin-wave Goldstone modes of the Skyrme crystal can relax nuclear spins. The (spatially averaged) relaxation rate has a Korringa temperature dependence,

$$
\frac{1}{T_{1}} \propto \frac{k_{\mathrm{B}} T}{\hbar \Omega} \sum_{\mathbf{G}} \int d^{2} k \chi_{+-}^{\prime \prime}(\mathbf{k}+\mathbf{G}, \mathbf{k}+\mathbf{G}, \Omega),
$$

where $\Omega$ is the nuclear resonance frequency. $\chi_{+-}^{\prime \prime}$ has contributions proportional [14] to $\delta\left(\hbar \Omega-\epsilon_{j}(\vec{k})\right)$ where $\epsilon_{j}(\vec{k})$ is one of the collective mode dispersions shown in Fig. 1. At long wavelengths neither phonon nor 
spin-wave excitations change the total electronic spin $S_{z}$ so that there is no contribution to $T_{1}^{-1}$ from the $\mathbf{G}=0$ term in Eq. (4). However, both change $S_{z}$ locally, and contribute $\mathbf{G} \neq 0$ terms to Eq. (4) which can be extracted from our TDHFA calculations. We find that [14]

$$
\begin{aligned}
t_{\mathrm{sw}}^{-1} & =\frac{|\nu-1| X_{\mathrm{sw}}\left(\hbar \omega_{c}\right)^{2}}{4 \pi U J}, \\
t_{\mathrm{ph}}^{-1} & =\frac{2 X_{\mathrm{ph}}\left(\hbar \omega_{c}\right)^{2}}{3\left[4 \pi|\nu-1| \hbar \Omega k_{B} T_{M}\left(e^{2} / \epsilon \ell\right)\right]^{2 / 3}},
\end{aligned}
$$

where $t_{\mathrm{sw}}^{-1}$ and $t_{\mathrm{ph}}^{-1}$ are the spin-wave and phonon contributions to the relaxation rate normalized to the Korringa expression for the same 2DES at $B=0$, and $X_{\mathrm{sw}}$ and $X_{\mathrm{ph}}$ are numerical constants. [For $\nu=1.10$ and $\tilde{g}=0.010\left(e^{2} / \ell\right)$ our detailed numerical calculations yield $X_{\mathrm{sw}}=13$ and $X_{\mathrm{ph}}=1.9$.] We expect that, in practice, disorder pinning of the Skyrmion lattice will gap the phonon spectrum and suppress $t_{\mathrm{ph}}^{-1}$. The linear relationship we find between $t_{\mathrm{sw}}^{-1}$ and $|\nu-1|$ is in agreement with experiment. Using parameter values from Fig. 2, Eq. (5) implies that for $|\nu-1|=0.1$, the spin-relaxation rate should typically be $\sim 10^{3}$ times faster than for a $2 \mathrm{D}$ Fermi gas system at $B=0$, in rough quantitative agreement with the experimental results of Barrett et al. $[9,14]$. This enormous enhancement in $1 / T_{1}$ can bring nuclei into thermal equilibrium with the electrons and dramatically increase the apparent specific heat of the 2D electron system [10]. We speculate that the sharp peak in the apparent specific heat observed by Bayot et al. [10] may be associated with critical slowing down at the Skyrmion melting temperature which suppresses motional narrowing of the nuclear resonance in the well and thereby enhances thermal coupling to nuclei outside the quantum wells [20]. If so, the Skyrmion melting temperature at $|\nu-1| \sim 0.2$ is $\sim 30-40 \mathrm{mK}$, a believable $\sim 10$ times smaller than the naive KTHNY estimate.

This work was supported in part by NATO Collaborative Research Grant No. 930684, by the National Science Foundation under Grants DMR-9416906 and DMR-9503814, and by CICyT of Spain under Contract MAT 94-16906. HAF acknowledges the support of the A.P. Sloan Foundation and the Research Corporation. Helpful conversations with S. Barrett, Matthew Fisher, E. Fradkin, L. Martin-Moreno, K. Moon, J. Palacios, N. Read, S. Sachdev, S. Sondhi, C. Tejedor, and K. Yang are gratefully acknowledged.

[1] For an elementary discussion of the physics of quantum Hall ferromagnets, see S. M. Girvin and A. H. MacDonald, in Perspectives in Quantum Hall Effects, edited by Sankar Das Sarma and Aron Pinczuk (Wiley, New York, 1997).

[2] S. L. Sondhi, A. Karlhede, S.A. Kivelson, and E.H. Rezayi, Phys. Rev. B 47, 16419 (1993).

[3] D. H. Lee and C.L. Kane, Phys. Rev. Lett. 64, 1313 (1990).

[4] K. Moon, H. Mori, Kun Yang, S. M. Girvin, A. H. MacDonald, L. Zheng, D. Yoshioka, and Shou-Cheng Zhang, Phys. Rev. B 51, 5138 (1995).

[5] L. Brey, H. A. Fertig, R. Côté, and A.H. MacDonald, Phys. Rev. Lett. 75, 2562 (1995).

[6] H.A. Fertig, L. Brey, R. Côté, and A. H. MacDonald, Phys. Rev. B 50, 11018 (1994); H. A. Fertig, L. Brey, R. Côté, A. H. MacDonald, A. Karlhede, and S. Sondhi, Phys. Rev. B 55, 10671 (1997).

[7] A. G. Green, I. I. Kogan, and A. M. Tsvelik, Phys. Rev. B 54, 16838 (1996).

[8] S. Sachdev and T. Senthil, Report No. cond-mat/9602028 (unpublished).

[9] R. Tycko, S.E. Barrett, G. Dabbagh, L. N. Pfeiffer, and K. W. West, Science 268, 1460 (1995); S. E. Barrett, G. Dabbagh, L. N. Pfeiffer, K.W. West, and R. Tycko, Phys. Rev. Lett. 74, 5112 (1995).

[10] V. Bayot, E. Grivei, S. Melinte, M. B. Santos, and M. Shayegan, Phys. Rev. Lett. 76, 4584 (1996).

[11] A. H. MacDonald, H. A. Fertig, and Luis Brey, Phys. Rev. Lett. 76, 2153 (1996).

[12] C. Nayak and F. Wilczek, Phys. Rev. Lett. 77, 4418 (1996).

[13] M. P. A. Fisher, P. B. Weichman, G. Grinstein, and D. S. Fisher, Phys. Rev. B 40, 546 (1989).

[14] R. Côté, L. Brey, H. A. Fertig, A. H. MacDonald, S. M. Girvin, and H. T.C. Stoof (to be published).

[15] R. Côté and A.H. MacDonald, Phys. Rev. B 44, 8759 (1991); R. Côté, L. Brey, H. A. Fertig, and A. H. MacDonald, Phys. Rev. B 51, 13475 (1995); R. Côté and A. H. MacDonald, Phys. Rev. B 53, 10019 (1996).

[16] The approximations used by Green et al. (Ref. [7]), who argue against the occurrence of a square lattice Skyrme crystal, do not permit the spin-wave Goldstone mode energy to be estimated and result in phonon energies which are identical to those of point electrons. These approximations are too crude to meaningfully judge the stability of the square lattice state. While we cannot rigorously exclude other possible crystal structures in the strong orientation-dependent interaction regime, we have established that the square lattice is lower in energy than the most plausible competitive structures.

[17] See R. Morf, Phys. Rev. Lett. 43, 931 (1979) for a discussion of its applicability to the classical electron crystal.

[18] L.D. Landau and E. M. Lifshitz, Theory of Elasticity (Pergamon, Oxford, 1970), p. 37.

[19] The case of a Wigner crystal of point particles in a strong magnetic field is discussed by D. S. Fisher, Phys. Rev. B 26, 5009 (1982).

[20] S. E. Barrett (private communication). 